Pacific Journal of Mathematics

COMPLETELY ADEQUATE NEIGHBORHOOD SYSTEMS AND 


\title{
COMPLETELY ADEQUATE NEIGHBORHOOD SYSTEMS AND METRIZATION
}

\author{
SEAN B. O'ReILly
}

\begin{abstract}
In this paper, the notion of a completely adequate neighborhood system for a topological space is defined and used to obtain characterizations of discreteness and second countability. Certain conditions on the completely adequate neighborhood system are given which yield collection wise normality and paracompactness. The notion of a standardized topological space is introduced (the class of standardized spaces includes, among others, the separable spaces and the developable spaces) and the main theorem gives necessary and sufficient conditions for the metrizability of standardized spaces in terms of completely adequate neighborhood systems.
\end{abstract}

o. Introduction and preliminaries. A great deal of work has been done in the area of metrization criteria for $T_{1}$ topological spaces. Roughly speaking, these criteria generally fall into two broad categories which might be characterized as "indexed neighborhood" criteria and "covering" criteria. In this paper, an "unindexed neighborhood" criterion is developed, that is, a metrization criterion in which the set algebraic conditions which the neighborhoods of points must satisfy is separated from the indexing requirement.

Specifically, the set algebraic requirement is that the space have a linearly ordered, completely adequate neighborhood system (defined in $\S 1$, and the indexing requirement is that the space have a standardization (defined in $\S 2$ ).

In what follows, all spaces are assumed to be $T_{1}$. A "neighborhood system" will always mean a mapping, $\mathscr{C}$, which assigns to each $x \in X$ a neighborhood basis at $x$, denoted by $\mathscr{U}(x)$. For any subset, $A$, the closure of $A$ will be denoted by $\mathrm{Cl}[A]$, and its interior by Int $[A]$. Otherwise, notation and terminology will follow that of Kelley [3].

\section{Completely adequate neighborhood systems.}

DEFINITION 1.1. A completely adequate neighborhood system (henceforth abbreviated to C. A. system) is a map, $\mathscr{V}$, which assigns to each $x \in X$ a neighborhood basis, $\mathscr{Y}(x)$, at $x$, satisfying:

Given $x \in X$ and 0 open, with $x \in 0$, there exists an open set $N[x ; 0]$, with $x \in N[x ; 0] \subset 0$, and such that each $y \in N[x ; 0]$ has a neighborhood $V(y) \in \mathscr{V}(y)$ with the property that $N[x ; 0] \subset V(y) \subset 0$. 
$\mathscr{V}$ will be called linearly ordered (resp. well-ordered) if $\mathscr{V}(x)$ is linearly ordered (resp. well-ordered) by inclusion at each $x \in X$. Similarly, $\mathscr{V}$ will be called countable if $\mathscr{C}(x)$ contains only countably many distinct neighborhoods for each $x \in X$. Finally, it should be noted that there is no loss of generality in assuming that each $V(x) \in \mathscr{V}(x)$ is open, since the neighborhood system obtained by replacing each $V(x)$ by its interior is completely adequate as long as $\mathscr{V}$ is.

EXAMPles 1.2. I. Let $X$ be a metric space with metric $\rho$. Let $S(x, r)=\{y \in X \mid \rho(x, y)<r\}$, where $r$ is any positive real number. If $D$ is any set of positive real numbers with inf $[D]=0$, then we define, for each $x \in X, \mathscr{V}(x)$ by

$$
\mathscr{V}(x)=\{S(x, d) \mid d \in D\} .
$$

Now, if 0 is open and $x \in 0$, then there exists $d \in D$ such that $S(x, d) \subset 0$. Moreover, there exist $p, q \in D$ such that $p<d / 2$ and $q<p / 2$. It is easily checked that if we take $N[x ; 0]=S(x, q)$, then $N[x ; 0] \subset S(y, p) \subset 0$ for each $y \in N[x ; 0]$. Since $S(y, p) \in \mathscr{V}(y)$, it follows that $\mathscr{V}$ is a C. A. system. Note that, for any selection of $D, \mathscr{V}$ is a linearly ordered C. A. system. If $D$ is also well-ordered then $\mathscr{V}$ will be a well-ordered C. A. system. In particular, if $D=\{1 / n \mid n=1,2, \cdots\}$, then $\mathscr{V}$ will be a countable, well-ordered C. A. system.

II. Let $X$ be an arbitrary topological space and $\mathscr{B}$ a base for the topology on $X$. Define $\mathscr{U}(x)=\{0 \in \mathscr{B} \mid x \in 0\}$, for each $x \in X$. If $x$ is arbitrary and $U$ is any open set containing $x$, then there exists $0 \in \mathscr{B}$ such that $x \in 0 \subset U$. If we take $N[x ; U]=0=V(y)$, then it is apparent that

$$
N[x ; U] \subset V(y) \subset U
$$

for every $y \in N[x ; U]$, and hence, the map $\mathscr{V}: x \rightarrow \mathscr{Y}(x)$ is a C. A. system. Note that if $\mathscr{B}$ is countable (i.e. $X$ is second countable) then $\mathscr{V}$ is a countable C. A. system.

III. Let $X$ be a set, $\mathfrak{U}$ a uniform structure on $X, \mathscr{T}$ the associated uniform topology on $X$, and $\mathfrak{B}$ a basis for $\mathfrak{U}$. If $V \in \mathfrak{B}, V[x]$ denotes the set $\{y \mid(x, y) \in V\}$. For each $x \in X$, let $\mathscr{Y}(x)=\{V[x] \mid V \in \mathfrak{B}\}$. We will show that the map, $\mathscr{V}: x \rightarrow \mathscr{C}(x)$ is a C. A. system for $X$.

Let 0 be open and $x \in 0$. Since $\mathfrak{B}$ is a basis for $\mathfrak{U}$, there exists $W \in \mathfrak{B}$ such that $W[x] \subset 0$ Moreover, there exists $V \in \mathfrak{B}$ such that $V \circ V \subset W$ and there exists $N \in \mathfrak{U}$ such that $N=N^{-1}$ and $N \circ N \subset V$. Now, since $N \subset V \subset W$, it follows that $N[x] \subset W[x] \subset 0$. Let $y \in N[x]$. Since $N=N^{-1}$, it follows that $(y, x) \in N$. If $z$ is an arbitrary element 
of $N[x]$, then $(x, z) \in N$, so $(y, z) \in N \circ N \subset V$, or $z \in V[y]$. Hence, $N[x] \subset V[y]$ for each $y \in N[x]$. Next, if $w \in V[y]$, then we have that $(x, y) \in N \subset V$ and $(y, w) \in V$, so $(x, w) \in V \circ V \subset W$. Hence, $V[y] \subset W[x]$. We have now shown that $N[x] \subset V[y] \subset W[x] \subset 0$ for each $y \in N[x]$. Finally, take $N[x ; 0]=\operatorname{Int}[N[x]]$ and it follows that $\mathscr{Y}$ is a C. A. system.

It is easy to see that if the basis, $\mathfrak{B}$, is linearly ordered by inclusion (resp. well-ordered by inclusion), then so is $\mathscr{C}(x)$ for every $x \in X$. In this case, the associated map, of, is a linearly ordered (resp. well-ordered) C. A. system.

We now develop some of the basis properties of C. A. systems

Lemma 1.3. Let $X$ be a topological space and or a C. A. system for $X$. If $D$ is any dense subset of $X$, then the collection $\bigcup\{\mathscr{V}(d) \mid d \in D\}$ is a base for the topology on $X$.

Proof. Let $0 \subset X$ be open and let $x$ be an arbitrary element of 0 . Since $\mathscr{V}$ is a C. A. system, there exists an open set $N[x ; 0]$ satisfying the condition of the definition. Since $D$ is dense in $X$, $N[x ; 0] \cap D \neq \varnothing$ and, hence, there exists $d_{x} \in D \cap N[x ; 0]$ and $V\left(d_{x}\right) \in$ $\mathscr{V}\left(d_{x}\right)$ such that

$$
x \in N[x ; 0] \subset V\left(d_{x}\right) \subset 0 .
$$

Hence $0=\mathbf{U}\left\{V\left(d_{x}\right) \mid x \in 0\right\}$, and so $\mathbf{U}\{\mathscr{V}(d) \mid d \in D\}$ is a base for the topology.

Second countable spaces may now be characterized in terms of C. A. systems.

Theorem 1.4. $X$ is a second countable space iff $X$ is separable and has a countable C. A. system.

Proof. It is well known that second countability implies separability. If $\mathscr{B}$ is a countable base for the topology on $X$, then defining $\mathscr{Y}$ as in 1.2. II. yields a countable C. A. system.

Conversely, if $D$ is a countable dense subset of $X$, and $\mathscr{V}$ is a countable C. A. system, then $\mathscr{C}(d)$ is countable for each $d \in D$, and hence, $\mathbf{U}\{\mathscr{C}(d) \mid d \in D\}$ is countable. By $1.3 \mathbf{U}\{\mathscr{f}(d) \mid d \in D\}$ is a base for the topology, so $X$ is second countable.

Discrete spaces may also be characterized in terms of C. A. systems.

THEOREM 1.5. $X$ is discrete iff every neighborhood system for $X$ is a C. A. system.

Proof. Let $X$ be a discrete space and $\mathscr{U}$ an arbitrary neigh- 
borhood system for $X$. Since $\{x\}$ is open, it follows that $\{x\} \in \mathscr{U}(x)$. Hence, it is obvious that $\{x\}=N[x ; 0]$ satisfies the requirement in 1.1 for any open set 0 which contains $x$, so $\mathscr{U}$ is a C. A. system.

Conversely, if $x$ is a non-isolated point of $X$, and $\mathscr{U}$ is a neighborhood system for $X$, define $\mathscr{V}$ by

$$
\begin{aligned}
& \mathscr{V}(y)=\{U(y)-\{x\} \mid U(y) \in \mathscr{U}(y)\} \text { if } x \neq y, \text { and } \\
& \mathscr{V}(x)=\mathscr{U}(x) .
\end{aligned}
$$

Since $X$ is $T_{1}$, it follows that $\mathscr{V}$ is a neighborhood system for $X$, but since $x \notin V(y)$ for any $y \neq x, \mathscr{V}$ is not a C. A. system for $X$.

We now investigate the properties of linearly ordered and wellordered C. A. systems.

Lemma 1.6. If $X$ has a linearly ordered $C$. A. system then $X$ is collectionwise normal.

Proof. Let $\left\{F_{\alpha} \mid \alpha \in A\right\}$ be a discrete collection of closed subsets of $X$. For $\alpha \in A$, let $F_{\alpha}^{*}=\bigcup\left\{F_{\beta} \mid \beta \in A, \beta \neq \alpha\right\}$. Since the family $\left\{F_{\alpha} \mid \alpha \in A\right\}$ is discrete, $F_{\alpha}^{*}$ is closed and $F_{\alpha} \cap F_{\alpha}^{*}=\varnothing$. Hence, $X-F_{\alpha}^{*}$ is open, and $F_{\alpha} \subset X-F_{\alpha}^{*}$. Thus, for each $x \in F_{\alpha}$, there exists an open set, $N\left[x ; X-F_{\alpha}^{*}\right]$, with the property that each of its points, $y$, has a neighborhood $V(y) \in \mathscr{C}(y)$ satisfying

$$
N\left[x ; X-F_{\alpha}^{*}\right] \subset V(y) \subset X-F_{\alpha}^{*} .
$$

Hence, define

$$
G_{\alpha}=\bigcup\left\{N\left[x ; X-F_{\alpha}^{*}\right] \mid x \in F_{\alpha}\right\} \text {. }
$$

Since $G_{\alpha}$ is the union of open sets, it is open. Moreover, since $x \in N\left[x ; X-F_{\alpha}^{*}\right]$ for each $x \in F_{\beta}$, we have that $F_{\alpha} \subset G_{\alpha}$, for every $\alpha \in A$.

Now, suppose that $G_{\alpha} \cap G_{\beta} \neq \varnothing$, for some $\alpha, \beta \in A$, where $\alpha \neq \beta$. Let $z$ be any element of $G_{\alpha} \cap G_{\beta}$. This entails the existence of points $x \in F_{\alpha}$, and $y \in F_{\beta}$ such that $z \in N\left[x ; X-F_{\alpha}^{*}\right]$ and $z \in N\left[y ; X-F_{\beta}^{*}\right]$. But this holds only if there exist neighborhoods $U(z), V(z) \in \mathscr{Y}(z)$ satisfying $x \in N\left[x ; X-F_{\alpha}^{*}\right] \subset U(z) \subset X-F_{\alpha}^{*}$ and $y \in N\left[y ; X-F_{\beta}^{*}\right] \subset$ $V(z) \subset X-F_{\beta}^{*}$. Since, by hypothesis, $\mathscr{V}$ is linearly ordered, either $U(z) \subset V(z)$ or $V(z) \subset U(z)$. Since $F_{\alpha} \subset F_{\beta}^{*}$, it follows that $X-F_{\beta}^{*} \subset$ $X-F_{\alpha}$, and since $V(z) \subset X-F_{\beta}^{*}$ we get $V(z) \cap F_{\alpha}=\varnothing$. But, $x \in U(z) \cap F_{\alpha}$, so we conclude that $V(z) \subset U(z)$. However, $F_{\beta} \subset F_{\alpha}^{*}$, so that $X-F_{\beta} \supset X-F_{\alpha}^{*} \supset U(z)$, and thus $U(z) \cap F_{\beta}=\varnothing$. But this contradicts $y \in V(z) \cap F_{\beta}$. Hence, $G_{\alpha} \cap G_{\beta}=\varnothing$, and $X$ is collectionwise normal.

Our next result makes use of the following, which may be found 
in $[3, \mathrm{pp} .155-156]$.

Definition. An open cover, $\mathcal{O}$, of a topological space, $X$, is even iff there exists a neighborhood, $V$, of the diagonal of $X \times X$ such that the family $\{V[x] \mid x \in X\}$ refines $\mathscr{O}$.

THEorem. If $X$ is a regular space, then $X$ is paracompact iff every open cover of $X$ is even.

Lemma 1.7. If $X$ has a well-ordered $C$. A. system, then $X$ is paracompact.

Proof. Let $\mathcal{O}$ be an open cover of $X$, and let $\mathscr{Y}$ be a well-ordered C. A. system for $X$. For each $x \in X$, let

$$
\mathscr{L}(x)=\{V(x) \in \mathscr{C}(x) \mid V(x) \subset 0 \text { for some } 0 \in \mathscr{O}\} \text {. }
$$

Since $\mathscr{C}(x)$ is well-ordered by inclusion, $\mathscr{C}(x)$ contains a least element, $U^{*}(x)$. That is, $U^{*}(x) \supset V(x)$ for every $V(x) \in \mathbb{Z}(x)$. Now, let $D=$ $\bigcup\left\{(x, y) \mid y \in U^{*}(x), x \in X\right\}$. Trivially, $D[x]=\{y \mid(x, y) \in D\}=U^{*}(x) \subset 0$ for some $0 \in \mathcal{O}$, so the family $\{D[x] \mid x \in X\}$ refines $\mathcal{O}$. It remains to show that $D$ is a neighborhood of the diagonal, $\Delta$. First, $D \supset \Delta$ since $(x, x) \in\left\{(x, y) \mid y \in U^{*}(x)\right\}$ for each $x \in X$. Next, note that since $\mathscr{Y}$ is C. A. system, there exists open $N\left[x ; U^{*}(x)\right]$ such that $x \in N\left[x ; U^{*}(x)\right] \subset$ $U^{*}(x)$. We also have that, for each $y \in N\left[x ; U^{*}(x)\right]$, there exists $V(y) \in \mathscr{P}(y)$ such that

$$
N\left[x ; U^{*}(x)\right] \subset V(y) \subset U^{*}(x) .
$$

But, since $U^{*}(x) \subset 0$ for some $0 \in \mathcal{O}$, it follows that $V(y) \subset 0$, so $V(y) \in \mathbb{Z}(y)$ and, therefore, $V(y) \subset U^{*}(y)$. Hence, $N\left[x ; U^{*}(x)\right] \subset U^{*}(y)$ for each $y \in N\left[x ; U^{*}(x)\right]$. Now, if $(y, z) \in N\left[x ; U^{*}(x)\right] \times N\left[x ; U^{*}(x)\right]$ then, since $y \in N\left[x ; U^{*}(x)\right], N\left[x ; U^{*}(x)\right] \subset U^{*}(y)$ and we have $(y, z) \in$ $\{y\} \times U^{*}(y) \subset D$. Hence, it follows that

$$
(x, x) \in N\left[x ; U^{*}(x)\right] \times N\left[x ; U^{*}(x)\right] \subset D
$$

for each $x \in X$, so $O$ is even.

Finally, we note that since of is well-ordered, it is linearly ordered, and thus $X$ is collectionwise normal by 1.6. $X$ is $T_{1}$, so it is regular and the result follows.

If $Y$ is any subspace of $X$ and $\mathscr{Y}$ is a C. A. system for $X$, define $\mathscr{Y}_{Y}$ by

$$
\mathscr{V}_{Y}(y)=\{V(y) \cap Y \mid V(y) \in \mathscr{Y}(y)\}
$$

for each $y \in Y$. It is a routine matter to verify that $\mathscr{V}_{Y}$ is a C. A. system for $Y$ with the relative topology. Moreover, it is clear that 
if $\mathscr{V}$ is linearly ordered (resp. well-ordered) then so is $\mathscr{V}_{Y}$. This remark, along with the two preceding results supply the proof of the next result.

THEOREM 1.8. If $X$ has a linearly ordered (resp. well-ordered) C. A. system then $X$ is hereditarily collectionwise normal (resp. hereditarily paracompact).

It was noted in 1.2. III that if $X$ has a uniform structure with a basis which is linearly ordered by inclusion, then $X$ also has a linearly ordered C. A. system and hence, by 1.8, $X$, with the associated uniform topology, is hereditarily collectionwise normal. In this case, however, the result can be improved.

THEOREM 1.9. Let $X$ be a uniform space with uniformity $\mathfrak{H}$. If $\mathfrak{u}$ has a basis which is linearly ordered by inclusion, then $X$, with the associated uniform topology is hereditarily paracompact.

Proof. Let $\mathfrak{W}$ be a linearly ordered basis for $\mathfrak{u}$. By means of a standard argument involving Zorn's Lemma, it is easy to show that $\mathfrak{B}$ contains a cofinal, well-ordered subfamily, $\mathfrak{B}$. Since $\mathfrak{B}$ is cofinal in $\mathfrak{W}$, it is easily verified that $\mathfrak{B}$ is a basis for $\mathfrak{U}$. Hence, as noted in 1.2. III, the associated map, $\mathscr{C}$, is a well-ordered C. A. system for $X$, so the result follows from 1.8 .

We are now in a position to give some metrization criteria for certain classes of topological spaces.

THeOREM 1.10. Let $X$ be a separable space. The following conditions are equivalent:

(a) $X$ is metrizable,

(b) $X$ has a countable, well-ordered C. A. system, and

(c) $X$ has a countable C. A. system and a linearly ordered $C$. A. system.

Proof. (a) implies (b): This was done in 1.2. I. (b) implies (c): Trivial. (c) implies (a): From 1.6 we have that $X$ is collectionwise normal. Since $X$ is $T_{1}$, it follows that $X$ is regular. From 1.4 we have that $X$ is second countable, so $X$ is metrizable by Urysohn's theorem [3, p 125].

Definition. A topological space, $X$, is developable if there exists a sequence of open coverings of $X,\left\{\mathscr{B}_{n} \mid n=1,2, \cdots\right\}$, such that $\left\{\operatorname{St}\left(x, \mathscr{B}_{n}\right) \mid n=1,2, \cdots\right\}$ is a neighborhood basis at $x$, where $\operatorname{St}\left(x, \mathscr{B}_{n}\right)=$ $\bigcup\left\{0 \mid x \in 0 \in \mathscr{B}_{n}\right\}$. 
Theorem 1.11. Let $X$ be a developable topological space. The following conditions are equivalent:

(a) $X$ is metrizable,

(b) $X$ has a countable, well-ordered C. A. system, and

(c) $X$ has a linearly ordered C. A. system.

Proof. (a) implies (b): This was done in 1.2. I. (b) implies (c): Trivial. (c) implies (a): Bing [1] has shown that every collectionwise normal, developable space is metrizable. By 1.6, $X$ is collectionwise normal, so $X$ is metrizable.

On the basis of the above two theorems, it would seem natural to conjecture that having a countable, well-ordered C. A. system is sufficient, in general, to guarantee metrizability. However, the next example shows that this is not the case.

EXAMPLE 1.12. There exists a topological space, $X$, with a countable, well-ordered C. A. system, $\mathscr{V}$, (in fact, $V(x, n) \supset \mathrm{Cl}[V(x, n+1)]$ for each $V(x, n), V(x, n+1) \in \mathscr{V}(x)$ and each $x \in X$.) but which is not perfectly normal, and, hence, not metrizable.

Let $X$ be the real numbers and $Q$ the rational numbers. A topology is defined on $X$ as follows:

$O \subset X$ is open iff $O=O_{1} \cup O_{2}$, where $O_{1}$ is open in the ordinary (metric) topology on $X$, and $O_{2} \subset X-Q$. This example appears in [4], where it is shown that $X$ is not perfectly normal, so all that is necessary is to construct the required C. A. system.

Let $V(x, n)=\{y \mid d(x, y)<1 / n\}$, where $d$ is the ordinary metric on $X$. Now, let

$$
\begin{aligned}
& \mathscr{Y}(x)=\{V(x, n) \mid n=1,2, \cdots\}, \text { if } x \in Q, \text { and } \\
& \mathscr{V}(x)=\{V(x, n) \mid n=1,2, \cdots, \omega\}, \text { if } x \in X-Q,
\end{aligned}
$$

where $V(x, \omega)=\{x\}$.

It is clear that the map $\mathscr{Y}: x \rightarrow \mathscr{V}(x)$ is a neighborhood system for $X$. To see that $\mathscr{C}$ is completely adequate, let $x \in X$ be arbitrary and $O \subset X$ be open, with $x \in O$.

If $x \in X-Q$, then $\{x\}$ is open and, hence, taking $\{x\}=N[x ; O]=$ $V(y, \omega)$, we have $N[x ; O] \subset V(y, \omega) \subset O$, for every $y \in N[x ; O]$.

If $x \in Q$, then, since $O=O_{1} \cup O_{2}$, where $O_{2} \subset X-Q$, we have $x \in O_{1}$, and $O_{1}$ is open in the ordinary topology. Therefore, there exists $M$ such that $V(x, M) \subset O_{1} \subset O$. Taking $N[x ; O]=V(x, 4 M)$, we have $N[x ; O] \subset V(y, 2 M) \subset O$, for each $y \in N[x ; O]$, and $\mathscr{y}$ is a C. A. system having the required properties.

2. Standardizations. In the light of 1.12 it is clear that some sort of additional condition is needed in order that a space with a 
suitable C. A. system be metrizable. From 1.10 and 1.11 it appears that any such property ought to be a common generalization of separability and developability. One condition that satisfies these requirements is that the space have a standardization.

DEFINITION 2.1. A standardization of a topological space, $X$, is a map, $\mathscr{K}$, which assigns to each $x \in X$ a countable, nested family of open sets, $\{U(x, n) \mid n=1,2,, \cdots\}$, such that

(a) $x \in U(x, n)$ for each $x \in X$ and $n=1,2, \cdots$.

(b) If $\left\{z_{n} \mid n=1,2, \cdots\right\}$ is a sequence of distinct points, then $\operatorname{Int}\left[\cap\left\{U\left(z_{n}, n\right) \mid n=1,2, \cdots\right\}\right]=\varnothing$.

(c) Int $[\cap\{U(x, n) \mid n=1,2, \cdots\}]=\{x\}$ if $x$ is isolated and is empty otherwise.

Included in the following examples are proofs that having a standardization is a common generalization of separable and developable spaces.

EXAMPLE 2.2. Let $X$ be a metric space with metric, $d$. If we let $\mathscr{C}(x)=\{S(x, n) \mid n=1,2, \cdots\}$, where, as usual,

$$
S(x, n)=\{y \mid d(x, y)<1 / n\},
$$

then it is easy to see that the map $\mathscr{U}: x \rightarrow \mathscr{U}(x)$ is a standardization.

Example 2.3. Let $X$ be a separable space, with $D$ a countable, dense subset of $X$. Let $\left\{d_{1}, d_{2}, \cdots\right\}$ be an enumeration of $D$. If $x$ is an isolated point of $X$, let $U(x, 1)=U(x, 2)=\cdots=\{x\}$. If $x$ is not isolated, let

$$
\begin{aligned}
& U(x, n)=X-\left\{d_{i} \mid i=1,2, \cdots, n\right\} \text { if } x \notin D, \text { and } \\
& U(x, n)=X-\left\{d_{i} \mid i=1,2, \cdots, n ; i \neq j\right\} \text { if } x=d_{j} \in D .
\end{aligned}
$$

It is clear that $x \in U(x, n)$ and that $U(x, n)$ is open for each $x \in X$ and $n=1,2, \cdots$. Furthermore, $\mathscr{U}(x)=\{U(x, n) \mid n=1,2, \cdots\}$ is nested and countable for each $x \in X$.

If $\left\{z_{i} \mid i=1,2, \cdots\right\}$ is a sequence of distinct points and $d_{k}$ is an arbitrary element of $D$, then $d_{k}$ is equal to at most one of the $z_{i}$, say $z_{j}$. Let $N=\max \{k, j+1\}$. It follows that $d_{k} \neq z_{N}$ and hence $d_{k} \notin U\left(z_{N}, N\right)$. Therefore, $d_{k} \notin \bigcap\left\{U\left(z_{i}, i\right) \mid i=1,2, \cdots\right\}$. Since $d_{k}$ was arbitrary, it follows that no element of $D$ is in $\bigcap\left\{U\left(z_{i}, i\right) \mid i=1,2, \cdots\right\}$ and hence Int $\left[\cap\left\{U\left(z_{i}, i\right) \mid i=1,2, \cdots\right\}\right]=\varnothing$.

Finally, if $x$ is an isolated point of $X$, from the definition of $U(x, n)$ we have that $\bigcap\{U(x, n) \mid n=1,2, \cdots\}=\{x\}$, so

$$
\text { Int }[\cap\{U(x, n) \mid n=1,2, \cdots\}]=\{x\} \text {. }
$$


Example 2.4. Let $X$ be a developable space. For each $x \in X$, let $O(x, n)$ be any open set satisfying $x \in O(x, n) \in \mathscr{B}_{n}$. Then, let $U(x, n)=$ $\bigcap\{O(x, i) \mid i=1,2, \cdots, n\}$. It is clear that $x \in U(x, n)$ and $U(x, n)$ is open for each $n=1,2, \cdots$ and each $x \in X$. Also, the family, $\mathscr{U}(x)=$ $\{U(x, n) \mid n=1,2, \cdots\}$ is nested and countable for each $x \in X$. Note that $U(x, n) \subset O(x, n) \subset \mathrm{St}\left(x, \mathscr{B}_{n}\right)$ for each $n=1,2, \cdots$ and each $x \in X$, so $\mathscr{Q}(x)$ is also a neighborhood basis at $x$.

If $\left\{z_{i} \mid i=1,2, \cdots\right\}$ is an arbitrary sequence of (not necessarily distinct) points of $X$, suppose that $x \in \operatorname{Int}\left[\cap\left\{U\left(z_{i}, i\right) \mid i=1,2, \cdots\right\}\right]$. We have, for each $n, x \in U\left(z_{n}, n\right) \subset O\left(z_{n}, n\right) \in \mathscr{\mathscr { B }}_{n}$. So,

$$
O\left(z_{n}, n\right) \subset \operatorname{St}\left(x, \mathscr{B}_{n}\right) \text {. }
$$

Thus,

$x \in \operatorname{Int}\left[\cap\left\{U\left(z_{n}, n\right) \mid n=1,2, \cdots\right\}\right] \subset \bigcap\left\{O\left(z_{n}, n\right) \mid n=1,2, \cdots\right\} \subset$

$$
\bigcap\left\{\operatorname{St}\left(x, \mathscr{B}_{n}\right) \mid n=1,2, \cdots\right\}=\{x\},
$$

the last equality from the fact that $\left\{\operatorname{St}\left(x, \mathscr{B}_{n}\right) \mid n=1,2, \cdots\right\}$ is a neighborhood basis at $x$, and that $X$ is $T_{1}$. We conclude that $\{x\}=$ Int $\left[\cap\left\{U\left(z_{n}, n\right) \mid n=1,2, \cdots\right\}\right]$, so $x$ is isolated. From this it follows that there exists $N$ such that $\{x\}=\operatorname{St}\left(x, \mathscr{B}_{n}\right)$ for every $n \geqq N$. From above, we have $O\left(z_{n}, n\right) \subset \operatorname{St}\left(x, \mathscr{B} \mathscr{S}_{n}\right)=\{x\}$, so $z_{n}=x$, for $n \geqq N$, and therefore, the sequence $\left\{z_{n} \mid n=1,2, \cdots\right\}$ is not composed of distinct points.

Finally, since $\mathscr{C}(x)$ is a neighborhood basis at $x$, we have $\{x\}=$ $\bigcap\{U(x, n) \mid n=1,2, \cdots\}$, so Int $[\bigcap\{U(x, n) \mid n=1,2, \cdots\}]=\{x\}$ iff $x$ is isolated and is empty otherwise.

The following sequence of results will be useful in the proof of the main theorem.

Lemma 2.5. Let $\mathscr{Q}$ be a standardization for $X$. Then the map, $\mathscr{W}$, defined by

$$
\begin{aligned}
& \mathscr{W}(x)=\{\{x\}\}, \text { if } x \text { is isolated, and } \\
& \mathscr{W}(x)=\mathscr{U}(x) \text { otherwise, }
\end{aligned}
$$

is also a standardization.

Proof. The proof is routine, and will be omitted.

Lemma 2.6. Let $\mathscr{Q}$ be a standardization for $X$, and let $O$ be any open set consisting of more than one point. Then, there exists $N$ such that $O \not \subset U(y, n)$ for any $y \in X$ and $n \geqq N$.

Proof. If not, then there exists a sequence of points, $\left\{z_{n} \mid n=\right.$ 
$1,2, \cdots\}$ such that $O \subset U\left(z_{n}, n\right), n=1,2, \cdots$. Now, if $\left\{z_{n} \mid n=1,2, \cdots\right\}$ contains a subsequence of distinct points, $\left\{z_{n_{k}} \mid k=1,2, \cdots\right\}$, then, since $n_{k} \geqq k$, we have $O \subset U\left(z_{n_{k}}, n_{k}\right) \subset U\left(z_{n_{k}}, k\right)$, so that $O \subset \bigcap\left\{U z_{n_{k}}, k\right) \mid k=$ $1,2, \cdots\}$, and hence $O \subset \operatorname{Int}\left[\bigcap\left\{U\left(z_{n_{k}}, k\right) \mid k=1,2, \cdots\right\}\right]$, which is a contradiction. Hence, $\left\{z_{n} \mid n=1,2, \cdots\right\}$ contains a constant subsequence, $z_{n_{k}}=x$ for some $x \in X$. But, this entails $O \subset U(x, n)$ for $n=1,2, \cdots$, since $O \subset U\left(x, n_{k}\right) \subset U(x, n)$ for each $n \leqq n_{k}$. We conclude that $O \subset \bigcap\{U(x, n) \mid n=1,2, \cdots\}$ and, since $O$ is open, that

$$
O \subset \operatorname{Int}[\cap\{U(x, n) \mid n=1,2, \cdots\}] \text {. }
$$

But this happens only if $O=\{x\}$, contradicting the assumption that $O$ consists of two or more points, completing the proof.

LEMma 2.7. Let $\mathscr{Q}$ be a standardization for $X$ and let $\mathscr{Y}$ be a linearly ordered neighborhood system-i.e. each $\mathscr{Y}(x)$ is linearly ordered by inclusion. Then, $X$ has a neighborhood system, $\mathscr{W}$, such that

(a) $\mathscr{\mathscr { C }}(x)=\{W(x, n) \mid n=1,2, \cdots\}$ is linearly ordered by inclusion, and

(b) if $V(x) \in \mathscr{V}(x)$ and $V(x) \subset U(y, n)$ for some $y \in X$, then $V(x) \subset$ $W(x, n)$.

Proof. Let $W(x, n)=\mathbf{U}\{V(x) \in \mathscr{Y}(x) \mid V(x) \subset U(y, n)$, for some $y \in X\}$, and let $\mathscr{W}(x)=\{W(x, n) \mid n=1,2, \cdots\}$. Note that (b) follows trivially. Next, if $m>n$, then $U(y, m) \subset U(y, n)$ for each $y \in X$, so that if $V(x) \subset U(y, m)$, then $V(x) \subset U(y, n)$ and so $W(x, m) \subset W(x, n)$, and (a) holds. It remains to be shown that $\mathscr{W}(x)$ is a neighborhood basis at each $x \in X$.

First, suppose that $x$ is isolated. By 2.5, we may assume that $\mathscr{C}(x)=\{\{x\}\}$. Now, by an argument similar to that of 2.6 , it can be shown that there exists $N$ such that $x \notin U(y, n)$ for any $y \neq x$ and $n \geqq N$. Thus, if $n \geqq N$ and $V(x) \subset U(y, n)$, it follows that $y=x$ and $U(y, n)=\{x\}$. Hence, $V(x)=\{x\}$, so $W(x, n)=\{x\}$ and $\mathscr{W}(x)$ is a neighborhood basis at $x$.

If $x$ is not isolated and $O$ is any open set containing $x$, let $V(x) \in \mathscr{Y}(x)$ satisfy $V(x) \subset O$. Since $x$ is not isolated, $V(x)$ consists of at least two points and therefore, by 2.6, there exists $N$ such that $V(x) \not \subset U(y, n)$ for any $y \in X$ and $n \geqq N$. Thus, if $S(x) \in \mathscr{C}(x)$ and $S(x) \subset U(y, n)$ for some $n \geqq N$, it follows that $S(x) \subset V(x)$ since $\mathscr{P}(x)$ is linearly ordered by inclusion. Thus, $W(x, n) \subset V(x) \subset O$ for each $n \geqq N$. Note that for each $x \in X$ and $n=1,2, \cdots, W(x, n) \neq \varnothing$ since there always exists $V(x) \in \mathscr{Y}(x)$ satisfying $x \in V(x) \subset U(x, n)$. This completes the proof.

It is well known that if a space is first countable then it has a 
neighborhood system which is linearly ordered at each point. 2.7 provides the following partial converse.

Corollary 2.8. Let $X$ have a standardization. If $X$ has a neighborhood system linearly ordered at each point, then $X$ is first countable.

Proof. The neighborhood system, $\mathscr{W}$, defined in 2.7 is countable at each point, so $X$ is first countable.

3. Metrization. We are now in a position to prove the main theorem.

THEOREM 3.1. A topological space is metrizable iff it has a linearly ordered C. A. system and a standardization.

Proof. If $X$ is metrizable then the required C. A. system was obtained in 1.2. I. and the standardization in 2.2.

Conversely, let $\mathscr{Q}$ be a standardization for $X$ and $\mathscr{O}$ a linearly ordered C. A. system. Let $S(x, n)=N[x ; U(x, n)]$, where $U(x, n) \in \mathscr{U}(x)$ and $N[x ; U(x, n)]$ is as in 1.1. Let $\mathscr{B}_{n}=\{S(x, n) \mid n=1,2, \cdots, x \in X\}$. It will be shown that $\left\{\mathscr{B}_{n} \mid n=1,2, \cdots\right\}$ is a development for $X$.

Let $x \in X$ be arbitrary and let $O$ be open, with $x \in O$. By hypothesis $\mathscr{C}$ is a linearly ordered C. A. system, so there exists a neighborhood system, $\mathscr{W}$, satisfying the conditions (a) and (b) of 2.7. Hence, there exists $m$ such that $W(x, m) \subset O$. Now, suppose that $x \in S(y, m)$ for some $y \in X$. This entails the existence of $V(x) \in \mathscr{V}(x)$ such that

$$
N[y ; U(y, m)]=S(y, m) \subset V(x) \subset U(y, m) .
$$

However, since $V(x) \subset U(y, m)$, it follows that $V(x) \subset W(x, m)$ by 2.7 . Thus, we have shown that if $x \in S(y, m)$ then

$$
S(y, m) \subset V(x) \subset W(x, m) \subset O,
$$

so St $\left(x, \mathscr{B}_{m}\right) \subset O$, and hence, since $O$ was arbitrary, $\left\{\operatorname{St}\left(x, \mathscr{B}_{n}\right) \mid n=\right.$ $1,2, \cdots\}$ is a neighborhood basis at $x$. Thus, $X$ is a developable space and therefore, by 1.11 , is metrizable.

In the light of $3.1,1.10$ and 1.11 may now be generalized as follows.

THEOREM 3.2. Let $X$ be a space with a standardization. The following conditions are equivalent:

(a) $X$ is metrizable, 
(b) $X$ has a countable, well-ordered C. A. system, and

(c) $X$ has a linearly ordered C. A. system.

In closing, we remark that a subsequent paper will deal with the situation that arises when one weakens the requirement " $\cdots N[x ; 0] \subset$ $V(y) \subset 0 \cdots "$ of 1.1 to " $\cdots x \in V(y) \subset 0 \cdots "$.

\section{REFERENCES}

1. R. H. Bing, Metrization of topological spaces, Canad. J. Math., 3 (1951), 105-125.

2. F. B. Jones, Metrization, Amer. Math. Monthly 73 (1966), 571-576.

3. J. L. Kelley, General Topology, New York, Van Nostrand 1955.

4. E. A. Michael, The product of a normal space and a metric space need not be normal, Bull. Amer, Math. Soc., 69 (1963), 375-376.

5. J. Nagata, A contribution to the theory of metrization, Journal of the Institute of Polytechnics, Osaka City University 8 (1957) pp. 185-192.

6. A. H. Stone, Sequences of coverings, Pacific J. Mathematics 10 (1960), 689-691.

Received January 26, 1971 and in revised form July 26, 1971. This paper forms a portion of the author's $\mathrm{Ph}$. D. dissertation, done at the University of Rochester under the direction of A. H. Stone. The author wishes to express his gratitude to Professor Stone for his help, advice and encouragement. The author is also indebted to the National Science Foundation and the University of Oklahoma for the support he received from them, under their Research Participation program, during the summer of 1968 . when a portion of the work herein reported was completed.

NIAGARA UNIVERSITY 


\section{PACIFIC JOURNAL OF MATHEMATICS}

\section{EDITORS}

\author{
H. SAMELSON \\ Stanford University \\ Stanford, California 94305

\section{R. HoвBY} \\ University of Washington \\ Seattle, Washington 98105
}

\section{J. DugundJI}

Department of Mathematics University of Southern California Los Angeles, California 90007

\author{
RichaRd ARENS \\ University of California \\ Los Angeles, California 90024
}

\section{ASSOCIATE EDITORS}
E. F. BECKENBACH
B. H. NEUMANN
F. WOLF
K. YoshidA

\section{SUPPORTING INSTITUTIONS}

\author{
UNIVERSITY OF BRITISH COLUMBIA \\ CALIFORNIA INSTITUTE OF TECHNOLOGY \\ UNIVERSITY OF CALIFORNIA \\ MONTANA STATE UNIVERSITY \\ UNIVERSITY OF NEVADA \\ NEW MEXICO STATE UNIVERSITY \\ OREGON STATE UNIVERSITY \\ UNIVERSITY OF OREGON \\ OSAKA UNIVERSITY
}

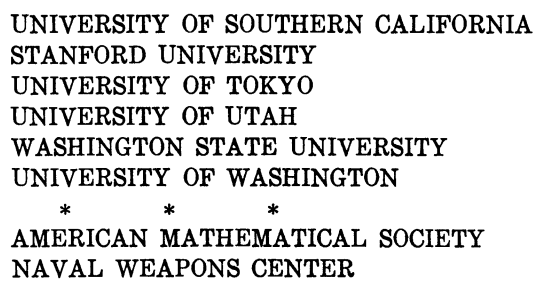

The Supporting Institutions listed above contribute to the cost of publication of this Journal, but they are not owners or publishers and have no responsibility for its content or policies.

Mathematical papers intended for publication in the Pacific Journal of Mathematics should be in typed form or offset-reproduced, (not dittoed), double spaced with large margins. Underline Greek letters in red, German in green, and script in blue. The first paragraph or two must be capable of being used separately as a synopsis of the entire paper. The editorial "we" must not be used in the synopsis, and items of the bibliography should not be cited there unless absolutely necessary, in which case they must be identified by author and Journal, rather than by item number. Manuscripts, in dup icate if possible, may be sent to any one of the four editors. Please classify according to the scheme of Math. Rev. Index to Vol. 39. All other communications to the editors should be addressed to the managing editor, Richard Arens, University of California, Los Angeles, California, 90024.

50 reprints are provided free for each article; additional copies may be obtained at cost in multiples of 50 .

The Pacific Journal of Mathematics is published monthly. Effective with Volume 16 the price per volume (3 numbers) is $\$ 8.00$; single issues, $\$ 3.00$. Special price for current issues to individual faculty members of supporting institutions and to individual members of the American Mathematical Society: $\$ 4.00$ per volume; single issues $\$ 1.50$. Back numbers are available.

Subscriptions, orders for back numbers, and changes of address should be sent to Pacific Journal of Mathematics, 103 Highland Boulevard, Berkeley, California, 94708.

PUBLISHED BY PACIFIC JOURNAL OF MATHEMATICS, A NON-PROFIT CORPORATION

Printed at Kokusai Bunken Insatsusha (International Academic Printing Co., Ltd.), 270, 3-chome Totsuka-cho, Shinjuku-ku, Tokyo 160, Japan. 


\section{Pacific Journal of Mathematics}

\section{Vol. 41, No. 2 December, 1972}

Tom M. (Mike) Apostol, Arithmetical properties of generalized Ramanujan sums .......................................... 281

David Lee Armacost and William Louis Armacost, On p-thetic groups ........ 295

Janet E. Mills, Regular semigroups which are extensions of groups .......... 303

Gregory Frank Bachelis, Homomorphisms of Banach algebras with minimal ideals ................................................ 307

John Allen Beachy, A generalization of injectivity .................. 313

David Geoffrey Cantor, On arithmetic properties of the Taylor series of rational functions. II.........................................

Václáv Chvátal and Frank Harary, Generalized Ramsey theory for graphs. III.

Small off-diagonal numbers .................................. 335

Frank Rimi DeMeyer, Irreducible characters and solvability of finite groups . . . . 347

Robert P. Dickinson, On right zero unions of commutative semigroups........ 355

John Dustin Donald, Non-openness and non-equidimensionality in algebraic

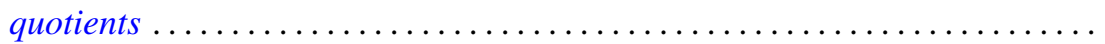

John D. Donaldson and Qazi Ibadur Rahman, Inequalities for polynomials with a prescribed zero ........................................ 375

Robert E. Hall, The translational hull of an $N$-semigroup ................ 379

John P. Holmes, Differentiable power-associative groupoids.............. 391

Steven Kenyon Ingram, Continuous dependence on parameters and boundary data for nonlinear two-point boundary value problems .

Robert Clarke James, Super-reflexive spaces with bases ..........

Gary Douglas Jones, The embedding of homeomorphisms of the plane in

continuous flows...............................

Mary Joel Jordan, Period $H$-semigroups and $t$-semisimple periodic

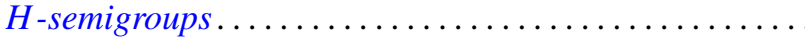

Ronald Allen Knight, Dynamical systems of characteristic 0

Kwangil Koh, On a representation of a strongly harmonic ring by sheaves...

Hui-Hsiung Kuo, Stochastic integrals in abstract Wiener space. ..

Thomas Graham McLaughlin, Supersimple sets and the problem of extending a

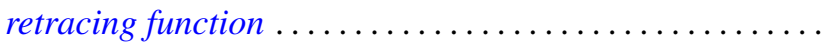

William Nathan, Open mappings on 2-manifolds .

M. J. O'Malley, Isomorphic power series rings

Sean B. O'Reilly, Completely adequate neighborhood systems and metrization

Qazi Ibadur Rahman, On the zeros of a polynomial and its derivative...

Russell Daniel Rupp, Jr., The Weierstrass excess function ..

Hugo Teufel, A note on second order differential inequalities and functional

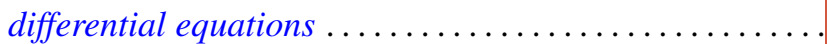

M. J. Wicks, A general solution of binary homogeneous equations over free 